\title{
Squeezing molecular thin alkane lubrication films between curved solid surfaces with long-range elasticity: Layering transitions and wear
}

Sivebæk, Ion Marius; Samoilov, V. N.; Persson, B. N. J.

Published in:

Journal of Chemical Physics

Link to article, DOI:

$10.1063 / 1.1582835$

Publication date:

2003

Document Version

Publisher's PDF, also known as Version of record

Link back to DTU Orbit

Citation (APA):

Sivebæk, I. M., Samoilov, V. N., \& Persson, B. N. J. (2003). Squeezing molecular thin alkane lubrication films between curved solid surfaces with long-range elasticity: Layering transitions and wear. Journal of Chemical Physics, 119(4), 2314-2321. https://doi.org/10.1063/1.1582835

\section{General rights}

Copyright and moral rights for the publications made accessible in the public portal are retained by the authors and/or other copyright owners and it is a condition of accessing publications that users recognise and abide by the legal requirements associated with these rights.

- Users may download and print one copy of any publication from the public portal for the purpose of private study or research.

- You may not further distribute the material or use it for any profit-making activity or commercial gain

- You may freely distribute the URL identifying the publication in the public portal 


\title{
Squeezing molecular thin alkane lubrication films between curved solid surfaces with long-range elasticity: Layering transitions and wear
}

\author{
I. M. Sivebaek \\ IFF, FZ-Jülich, 52425 Jülich, Germany and MEK-Energy, Technical University of Denmark, \\ 2800 Lyngby, Denmark \\ V. N. Samoilov \\ IFF, FZ-Jülich, 52425 Jülich, Germany and Physics Faculty, Moscow State University, \\ 117234 Moscow, Russia
}

B. N. J. Persson

IFF, FZ-Jülich, 52425 Jülich, Germany

(Received 28 February 2003; accepted 24 April 2003)

\begin{abstract}
The properties of alkane lubricants confined between two approaching solids are investigated by a model that accounts for the curvature and the elastic properties of the solid surfaces. We consider linear alkane molecules of different chain lengths, $\mathrm{C}_{3} \mathrm{H}_{8}, \mathrm{C}_{4} \mathrm{H}_{10}, \mathrm{C}_{8} \mathrm{H}_{18}, \mathrm{C}_{9} \mathrm{H}_{20}, \mathrm{C}_{10} \mathrm{H}_{22}, \mathrm{C}_{12} \mathrm{H}_{26}$, and $\mathrm{C}_{14} \mathrm{H}_{30}$ confined between smooth gold surfaces. In most cases we observe well defined molecular layers develop in the lubricant film when the width of the film is of the order of a few atomic diameters. An external squeezing-pressure induces discontinuous, thermally activated changes in the number $n$ of lubricant layers. We find that with increasing alkane chain length, the transition from $n$ to $n-1$ layers occurs at higher pressure, as expected based on the increasing wettability (or spreading pressure) with increasing chain length. Thus, the longer alkanes are better boundary lubricants than the shorter ones, and this should result in less wear. We obtain good correlation between our theoretical results and wear experiments. (C) 2003 American Institute of Physics. [DOI: 10.1063/1.1582835]
\end{abstract}

\section{INTRODUCTION}

Sliding friction and wear are two of the oldest problems in physics, and have undoubtedly a huge practical importance. ${ }^{1,2}$ In recent years, the development of technologically advanced devices has shown a need for durable low-friction surfaces and lubricant fluids. In these devices the interest is focused on the stability under pressure of thin lubricant films, since the complete squeeze out of the lubricant from an interface may give rise to cold-welded junctions, resulting in high friction and catastrophically large wear. Thus, for example, experiments have shown that two smooth mica surfaces separated by a molecular thin lubrication film will slide without wear, while huge wear occurs when the last lubrication monolayer has been squeezed out. ${ }^{3}$

Wear is not, normally, a major problem in devices equipped with a separate lubricating system as the lubricating fluid here can be improved to meet the demands of the actual system. However, in some cases, e.g., in a diesel engine injection pump, wear can be a significant problem. Modern injection pumps may operate at injection pressures of 2000 bars, and the devices rely on the lubricating abilities of the pumped fuel. As the fuel has to be burned in the engine, it cannot be refined to a very large extent. An excessive use of additives would be disadvantageous both for the environment and the economy.

The investigation of the lubrication abilities of fuels began in the 1960s when airplane injection pumps showed extensive wear as a result of lowering the sulphur content of the fuel. The same happened for diesel injection pumps in the 1980s as the sulphur content in diesel fuels was reduced for environmental reasons. The main issue in both cases is that sulphur containing species and other polar molecules bind to the surfaces chemically and they are thereby major contributors to pump lubrication. ${ }^{4}$ Currently, the boundary lubrication ability of low-sulphur fuels is improved by small amounts of antiwear agents.

The parameters describing the lubrication qualities of a diesel fuel are the viscosity and the lubricity. The viscosity is important in the hydrodynamic regime whereas the lubricity dominates in the boundary lubrication regime. As the viscosity of a diesel fuel is more than ten times lower than that of most lubricating oils, the boundary lubricating regime is predominant in injection pumps. As a result of the wear problems, lubricity measuring devices have been developed. The currently used test is the high-frequency reciprocating rig (HFRR), which is covered by several standards. 5,6

The HFRR is a laboratory test based on a ball on disk configuration as shown in Fig. 1. A steel ball oscillates on a steel disk, while the contact is only lubricated by the tested fuel. The ball has a given load, which is currently $2 \mathrm{~N}$. The other current test conditions are: Stroke $1 \mathrm{~mm}$, duration 75 min, temperature 25 or $60^{\circ} \mathrm{C}$, frequency of oscillations of the ball $50 \mathrm{~Hz}$, mean velocity $0.1 \mathrm{~m} / \mathrm{s}$. $^{5,6}$ The result of a HFRR test is a measure of the wear scar on the ball. The major and the minor axes of the wear scar are measured and the wear scar diameter (WSD) is expressed as the mean of the two 


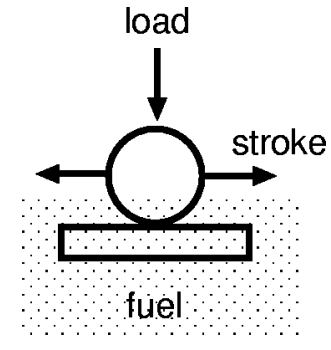

FIG. 1. Ball on disk configuration of a lubricity test. The current used test conditions are: Stroke $1 \mathrm{~mm}$, load $2 \mathrm{~N}$ and test duration $75 \mathrm{~min}$ (Refs. 5 and 6). Radius of steel ball $3 \mathrm{~mm}$. Ball oscillates with frequency $50 \mathrm{~Hz}$. Mean velocity $0.1 \mathrm{~m} / \mathrm{s}$.

axes given in microns. Currently, 460 microns is the pass limit for a fuel. ${ }^{6}$

Up to now, the HFRR test has mainly served as a tool for dosing lubricity additive to the fuel. Only a few parts per million of long polar molecules will raise the lubricity of a fuel considerably. Recently a pressurized lubricity test appeared that can handle dimethyl ether (DME), a new promising fuel for diesel engines. ${ }^{7}$ DME has a vapor pressure of about 6 bars at $25 \mathrm{C}$ and a very low viscosity so it resembles propane. By mixing DME with additives the lubricity is easily raised but a full-scale pump test reveals still too large wear when pumping additised DME. This observation coupled with an extensive statistical analysis of HFRR results indicate that the viscosity of the fuel may have a significant influence on the wear scar diameter. ${ }^{8}$ From these studies, it was concluded that a low viscosity fuel needs to have a higher lubricity than a similar high viscosity fuel in order to obtain acceptable lifetime of the injection pumps.

The relation between viscosity and wear scar diameter is not easily deducted from lubricity studies. A fuel is a mixture of alkanes of different lengths, branched molecules, aromatic molecules and some polar species. Each of these components has its own influence on the viscosity and lubricity of the fuel. One interesting factor is the influence of the length of a molecule on the amount of wear in the lubricity test. The reason for this is that low viscosity aviation fuels are composed mainly of molecules with 6-16 carbon atoms whereas the higher viscosity diesel fuels consist of hydrocarbons with 16-22 carbon atoms, ${ }^{9}$ which indicates a significant importance of the molecule length.

Figure 2 shows the dynamic viscosity of linear alkanes as a function of their length expressed as the number of carbon atoms in the molecule. ${ }^{10-12}$ It is obvious that longer alkanes have higher viscosities than the shorter ones. An experimental study has correlated the results from HFRR measurements and viscosities for a number of different pure substances, among them some linear alkanes. ${ }^{9}$ However, we do not believe that the viscosity is the most relevant variable in the present case; we will present an alternative explanation based on the nature of the lubricantwall interaction.

Figure 3(a) gives the lubricity of a number of linear alkanes as a function of the number of carbon atoms in the molecules. ${ }^{9}$ In Fig. 3(b), results from another recent experi-

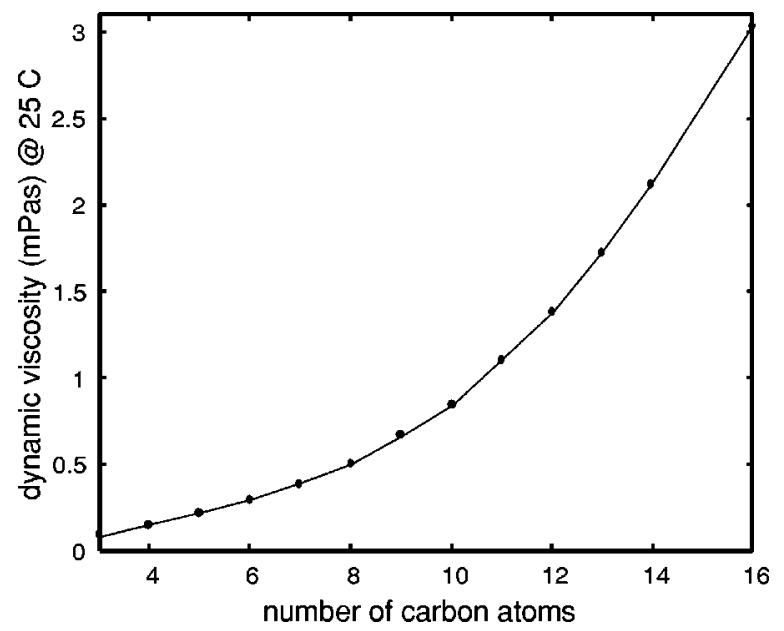

FIG. 2. The dynamic viscosity of linear alkanes at $25 \mathrm{C}$ as a function of the length of the molecules. The results for propane and butane ( 3 and 4 carbon atoms) were obtained at the vapor pressure of the liquids at $25 \mathrm{C}$ (Refs. 11 and 12). The other results were obtained at ambiant pressure (Ref. 10).

mental study are shown. ${ }^{11}$ As the lubricity test equipment was pressurised in this experiment, propane and butane could also be studied and are included in the figure. The lubricity levels in the two experimental studies are different due to differences in the test conditions, but in both cases the wear scar diameter decreases with increasing alkane length. The volume of steel worn off the ball is proportional to the wear scar diameter to the exponent four. Since the ratio of the wear scar diameters, when going from propane (3 carbon atoms) to hexadecane (16 carbon atoms) [see Fig. 3(b)], equals about 2, about 16 times larger steel volume has been worn off in the former case.

In this paper we will present computer simulations and analytical argument about the influence of linear hydrocarbons on wear. We will use a model developed for studying the atomistic origin of sliding friction, which accounts for long range elastic effects. ${ }^{13}$ The model describes the squeeze-out of lubricating layers between two curved surfaces as they are approaching each other. The squeeze-out of the last layer implies a huge increase in the wear as coldwelded junctions then can be formed.

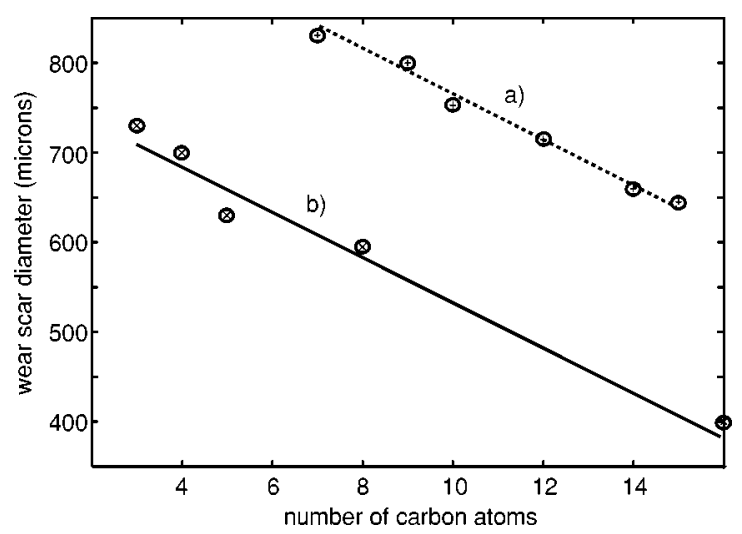

FIG. 3. The lubricity, expressed as the wear scar diameter, as a function of the molecule length for a number of linear alkanes. Results (a) are from Ref. 9 and (b) from Ref. 11. 


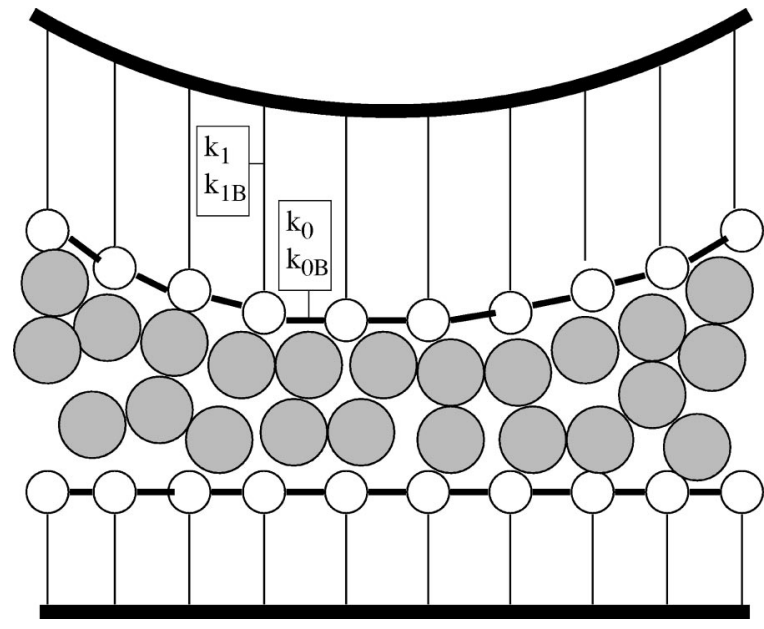

FIG. 4. Schematic picture of the central region of the squeezing model used in the present article.

\section{THE MODEL}

The model was described in Refs. 13 and 14, but we review it briefly here. We are concerned with the properties of a lubricant film squeezed between the curved surfaces of two elastic solids. In experiments, a system of this type is obtained by gluing two elastic slabs (of thickness $W_{1}$ and $W_{2}$ ) to "rigid" surface profiles of arbitrary shape. If the radii of curvature of the rigid surfaces are large compared to $W_{1}$ and $W_{2}$, the elastic slabs will deform, reproducing with their free surfaces the (nearly arbitrary) shape of the underlying rigid profiles.

In what follows we denote the lower solid as substrate, which is taken to be fixed in space. The upper solid, denoted as block, will be moving. To account for the elastic response of the slabs, without dealing with the large number of atoms required to simulate a mesoscopic elastic solid we treat explicitly, at the atomistic level, only the last atomic layer of the solids at the interface. These atoms are connected to a rigid curved surface (or profile). The force constants connecting these atoms to the rigid profile, however, are not the bare parameters, determined by the model interatomic potential. Instead, those force constants are treated as effective parameters that implicitly reintroduce the elastic response of the slabs of arbitrary width $W_{1}$ and $W_{2}$.

The model is illustrated in Fig. 4 (see also Refs. 13 and 14). The atoms in the bottom layer of the block (open circles) form a simple square lattice with lattice constant $a$, and lateral dimensions $L_{x}=N_{x} a$ and $L_{y}=N_{y} a$. In the following, periodic boundary conditions are assumed in the $x y$ plane. The atoms interact with each other via "stiff" springs (thick lines) and execute bending and stretching motion characterized by a bending force constant $k_{0 B}$ and a stretching force constant $k_{0}$, respectively. Moreover, each atom is connected to the upper rigid surface profile by "soft" elastic springs (thin lines), of bending force constant $k_{1 B}$ and stretching force constant $k_{1}$. As described in Refs. 13 and 14, the numerical values of all these force constants $k_{0}, k_{0 B}, k_{1}$, and $k_{1 B}$ are determined in such a way as to mimic the elastic response of the entire slab.
The substrate is treated in a similar way as the block, but we use slightly different lattice constant in order to avoid having (low order) commensurate structures formed at the interface. The space between the block and the substrate is occupied by a layer (monolayer or more) of the lubrication fluid (full circles in Fig. 4).

The MD (molecular dynamics) calculations have been performed by keeping the temperature of the solid walls fixed at their outer boundaries (see Ref. 13). This is a realistic treatment, and it implies that heat flows from the lubricant to the confining walls.

Below we study mainly the average pressure. The pressure acting on a wall atom is defined as the total normal force acting on the wall atom from the lubricant atoms and from the other wall, divided by the area $a^{2}$. The average pressure is the $z$-component of the total force acting on the solid block from the lubricant and the substrate, divided by the total area $L_{x} \times L_{y}$.

Below we provide details of the models used for the block, the substrate and the lubricants in the simulations carried out in the present work, which differ from those described in Refs. 13 and 14.

Both solids the block and the substrate were gold. We used the same elastic modulus and Poisson ratio for the block and substrate, which were $E=7.72 \cdot 10^{10} \mathrm{~Pa}$ and $\nu$ $=0.42$ for gold. We also used the same thickness for the block and substrate $W=50 \AA$. This choice of thicknesses imply that the block and the substrate used in our simulations will deform elastically similar to each other. In the simulations we used a system of lateral dimensions $L_{x}=506 \AA$, $L_{y}=75.9 \AA$. The block rigid profile was taken to be cosine corrugated in the $x$ direction, with corrugation amplitude (difference between maximal and minimal surface heights) $0.1 L_{x}$ and wavelength $L_{x}$. The substrate was atomically flat with $N_{x}=200$ and $N_{y}=30$ atoms in the $x$ and $y$ directions, forming a square lattice with lattice constant $a=2.53 \AA$. The corresponding parameters for the block were $N_{x}=180, N_{y}$ $=27$, and $a=2.81 \AA$.

Alkane lubricants were used in the present calculations. We considered alkane molecules of different chain lengths $\mathrm{C}_{3} \mathrm{H}_{8}, \mathrm{C}_{4} \mathrm{H}_{10}, \mathrm{C}_{8} \mathrm{H}_{18}, \mathrm{C}_{9} \mathrm{H}_{20}, \mathrm{C}_{10} \mathrm{H}_{22}, \mathrm{C}_{12} \mathrm{H}_{26}$, and $\mathrm{C}_{14} \mathrm{H}_{30}$. We considered $\mathrm{C}_{n} \mathrm{H}_{2 n+2}$ chain molecules consisting of $n$ beads in the united atom representation. The Lennard-Jones potential was used to model the interaction between beads of different chains

$$
v(r)=4 \epsilon_{0}\left[\left(\frac{r_{0}}{r}\right)^{12}-\left(\frac{r_{0}}{r}\right)^{6}\right]
$$

and the same potential with modified parameters $\left(\epsilon_{1}, r_{1}\right)$ was used for the interaction of each bead with the block and substrate atoms. For the interactions within the $\mathrm{C}_{n} \mathrm{H}_{2 n+2}$ we used the OPLS model (Refs. 15 and 16). The parameters were $\epsilon_{0}=5.12 \mathrm{meV}$ for both interior and end beads and $r_{0}$ $=3.905 \AA$ in all cases. Atomic mass 14 (for interior $\mathrm{CH}_{2}$ beads) and 15 (for the $\mathrm{CH}_{3}$ end groups) were used. For the interaction of each bead with the block and substrate atoms we took $\epsilon_{1}=18.60 \mathrm{meV}$ and $r_{1}=3.28 \AA .{ }^{17}$ The latter choice 


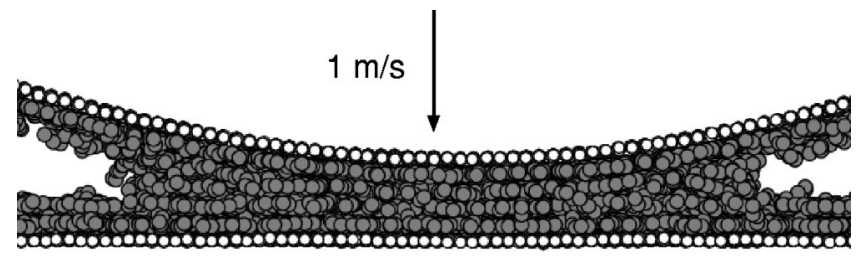

FIG. 5. Snapshot picture during squeezing with decane as lubricant. The squeezing velocity is $1 \mathrm{~m} / \mathrm{s}$. The block has moved a distance of $3 \AA$ resulting in a four monolayers thick film of decane between the surfaces.

reflects the stronger interaction between the beads and metal surfaces than between the bead units of different lubricant molecules.

Within a $\mathrm{C}_{n} \mathrm{H}_{2 n+2}$ chain we assume nearest neighbor $\mathrm{C}$ atoms are connected via springs with the spring constant $k$, which was chosen equal to $10 \mathrm{~N} / \mathrm{m}$. Time step was equal to 1 fs. We used an angle bending interaction of the form $E(\cos \theta) / k_{B}=1 / 2 k_{\text {bend }}\left(\cos \theta-\cos \theta_{0}\right)^{2}$ with $k_{\text {bend }}=62543 \mathrm{~K}$ and $\theta_{0}=2.0001 \mathrm{rad} .{ }^{16}$ For the dihedral interaction we used the functional form in term of a cosine Fourier series $E(\phi) / k_{B}=\sum_{i=0}^{3} c_{i} \cos ^{i}(\phi)$ with parameters $c_{0}=1009.99 \mathrm{~K}$, $c_{1}=2018.95 \mathrm{~K}, c_{2}=136.37 \mathrm{~K}, c_{3}=-3165.30 \mathrm{~K} .{ }^{16}$ Internal beads of separation greater than 3 units were treated similarly as beads from different chains.

For interaction between atoms of the block and the substrate we used the Lennard-Jones potential with the same parameters $\epsilon_{1}$ and $r_{1}$ as for the interaction of each bead with the block and substrate atoms.

Variation of the chain length of lubricant molecules gave a possibility to examine similarities and differences between squeezing behavior of lubricants consisting of short chain molecules (such as propane $\mathrm{C}_{3} \mathrm{H}_{8}$ ) and more complex lubricants (such as octane $\mathrm{C}_{8} \mathrm{H}_{18}$ or tetradecane $\mathrm{C}_{14} \mathrm{H}_{30}$ ).

\section{SIMULATION RESULTS}

In this section we describe the results obtained from our simulations for seven different linear alkanes as lubricants. Two cases are investigated: One with pure squeezing of the lubricant and another with simultaneous sliding and squeezing. In both cases, the velocities are $1 \mathrm{~m} / \mathrm{s}$, and the systems at the start of the squeezing are in thermal equilibrium at the temperature of $300 \mathrm{~K}$.

\section{A. Squeezing}

We consider the squeezing of seven alkanes starting from a point where the block and the substrate are separated by four monolayers of lubricant. The total squeezing distance is about $20 \AA$ depending on the considered system.

In order to illustrate the squeezing systems, decane $\left(\mathrm{C}_{10} \mathrm{H}_{22}\right)$ was chosen as example in Fig. 5. In this figure the arrow shows the squeezing movement of the block towards the substrate. It can be observed that the surfaces are separated by four monolayers of decane in the central region at a point where the block has moved a distance of $3 \AA$.

The main results of the computer simulations are shown in Fig. 6, where the average pressure during squeezing is plotted against the distance moved by the block. Of the seven investigated systems only propane, octane and tetradecane

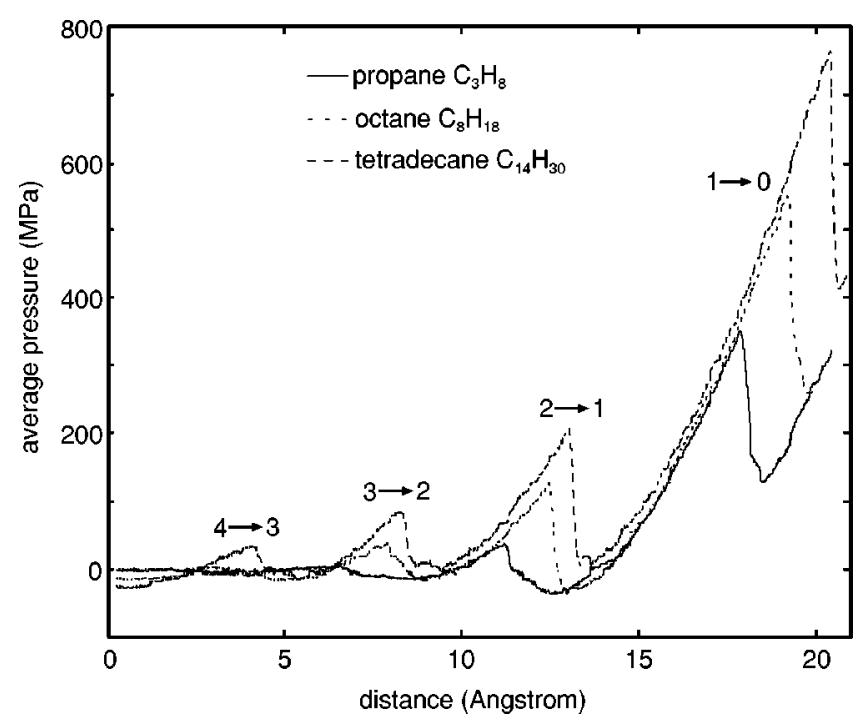

FIG. 6. The variation of the average pressure during squeezing developed as the block moves a distance of $20 \AA$ towards the substrate. The $n \rightarrow n-1$ layering transitions are shown for three systems: propane, octane and tetradecane.

are shown for clarity. Each jump in the pressure curves corresponds to a layering transition indicated by $n \rightarrow n-1$ in the figure. At about $20 \AA$ displacement, no lubricant is left between the surfaces and further movement of the block would only result in a monotone increase in pressure.

In Fig. 7 we show the pressure to initiate the layering transitions for all seven alkanes. Linear regression analysis is performed for each layering transition, and the resulting lines are also plotted in the figure.

\section{B. Squeezing and sliding}

The computer simulations with simultaneous squeezing and sliding have been performed with velocities of $1 \mathrm{~m} / \mathrm{s}$ in both the vertical and horizontal directions.

The systems are set up as shown in Fig. 8, illustrated by

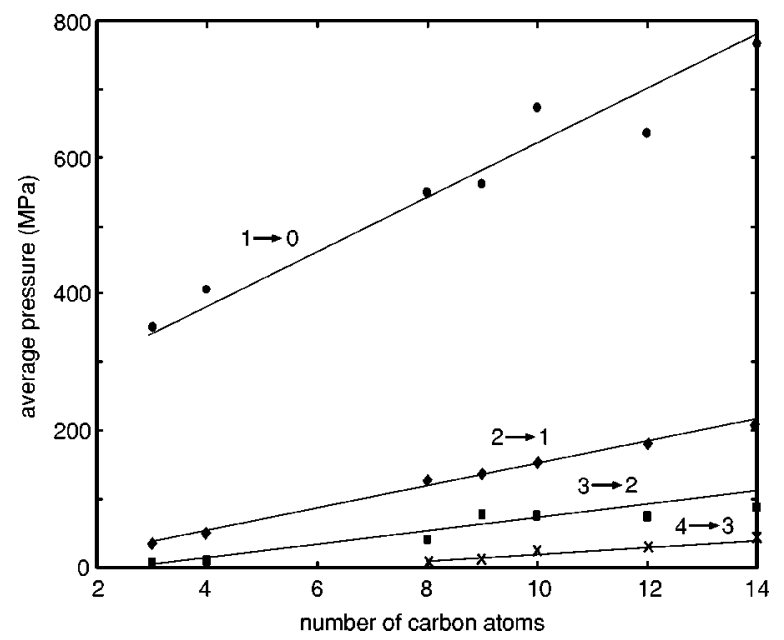

FIG. 7. The variation of the squeeze-out average pressure during squeezing as a function of the length of the alkane. The figure includes all the investigated alkanes. The straight lines are linear interpolation curves for the pressure at which the $n \rightarrow n-1$ layering transition occurs. 


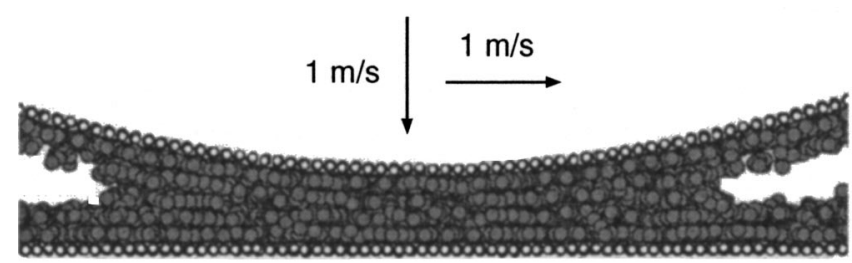

FIG. 8. Snapshot picture during squeezing and sliding with decane as lubricant. Both the squeezing and the sliding velocities are $1 \mathrm{~m} / \mathrm{s}$. The block has moved $3 \AA$ in the directions indicated by the arrows in this snapshot.

an example with decane as lubricant. Jumps corresponding to the $n \rightarrow n-1$ layering transitions are present in the curves showing the average pressure versus distance, just as it was in the case of squeezing only. The results are summarized in Fig. 9.

\section{DISCUSSION}

Let us first add a few comments to Fig. 6. As can be observed in this figure, the pressure is negative in the begining of the squeezing. This behavior has been observed earlier, ${ }^{14}$ and it is due to capillary bridge attraction as the lubricants are in a high-mobility, fluidlike state at this stage. During the remaining part of the squeezing, the lubricant behaves more solidlike and pressure build up precedes the squeeze out of the monolayers. Solidlike is here defined as the ability to sustain a normal (or tangential) stress which is not possible in the fluidlike state. The pressure also becomes negative after the first three monolayers squeeze-out, which is the result of a rebounding of the elastic surfaces after their compression during the pressure build up. For a macroscopic solid the pressure drop is very small, i.e., the negative pressure we observe is a result of the thin block used in the computer simulations.

Let us expand on the last point. A macroscopic block, say a steel cube with $L=1 \mathrm{~cm}$ side, at a typical squeezing pressure, say $P=1 \mathrm{MPa}$ (which is actually a low pressure), is compressed by a distance $d=L P / E=1000 \AA$ (where $E$

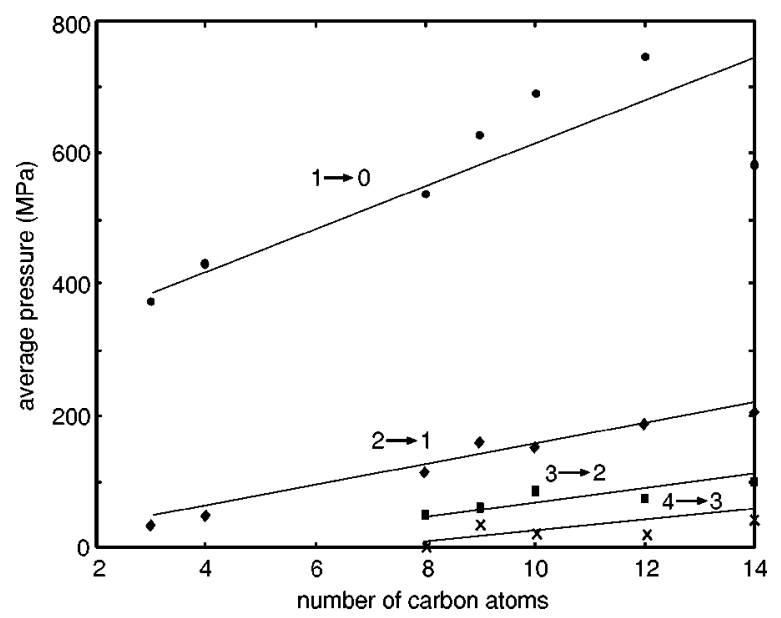

FIG. 9. The variation of the squeeze-out average pressure during squeezing and sliding as a function of the length of the alkane. The figure includes all the investigated alkanes. The straight lines are linear interpolation curves for the pressure at which the $n \rightarrow n-1$ layering transition occurs.
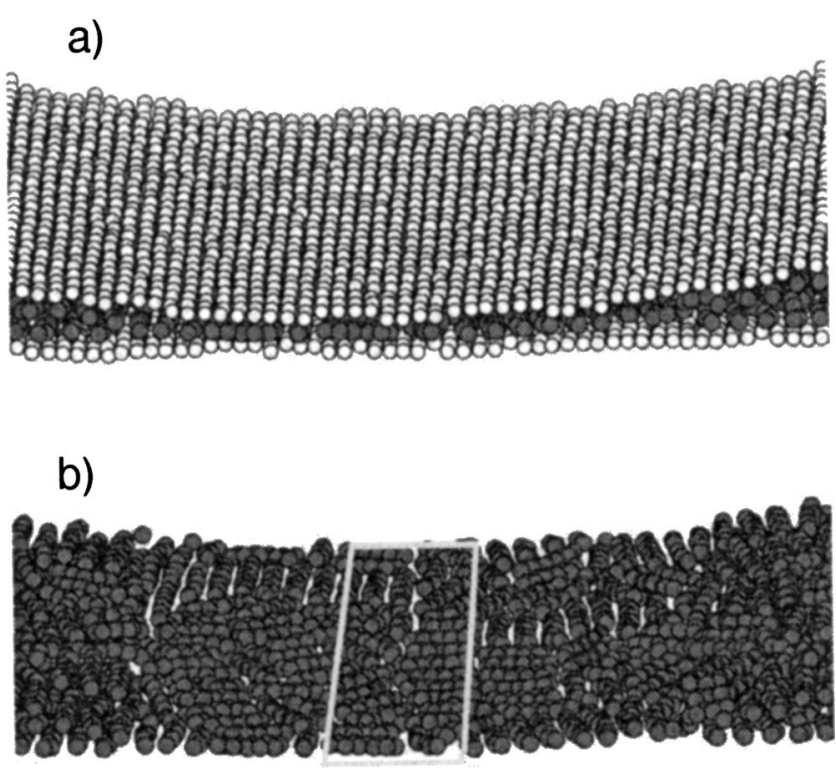

FIG. 10. The central part of the contact between the block and the substrate. In (a) the whole system is shown whereas in (b) the surface atoms have been removed and the section displayed in Fig. 11 is also shown. The width is the same as the width of the contact (75.9 $\AA$ ) whereas the length is $20 \AA$, which is $10 \AA$ on either side of the middle of the contact.

$=10^{11} \mathrm{~N} / \mathrm{m}^{2}$ is the elastic modulus). When one monolayer of lubricant is squeezed out the distance between the surfaces at the lubricant interface decreases by at most $10 \AA$ A. Thus, even if the upper surface of the block is kept fixed (clamped) the downward relaxation of the bottom surface of the block will give rise to a negligible change in the pressure in the block (the pressure drops by at most $1 \%$ ). On the other hand, in our case the block is only $\sim 100 \AA$ thick and when one monolayer gets squeezed out the pressure in the block, when the upper surface of the block is clamped [or, as in our case, forced to move very slowly (on the time scale of the squeeze-out) towards the substrate], typically decreases to nearly zero and it could even be negative.

From Figs. 6, 7, and 9, it is clear that longer alkanes are better boundary lubricants than the shorter ones. The squeeze-out of the lubricant layers, the last one in particular, occur at higher pressures and at smaller distances between the surfaces for long alkanes than for the short ones. In the present cases, there is very little difference between the results for squeezing only and the combined squeezing and sliding, as shown by Figs. 7 and 9. The reason for this is that the lubricant film is pinned to the bottom surface of the block and remains in a solidlike state during sliding. This differs from the earlier observations, ${ }^{13}$ where the squeeze-out of the last monolayer proved to be difficult, if not impossible, without a simultaneous sliding. When the sliding motion was applied, the squeeze-out of the last layer was quick and easy. The main difference between the earlier work and the present one, is that in the former case the (atomic) lubricant formed a commensurate and strongly pinned (to both walls) structure, which could not be removed by squeezing alone. However, a superimposed sliding motion "fluidized" the lubricant film, resulting in easy squeeze-out. In the present work 

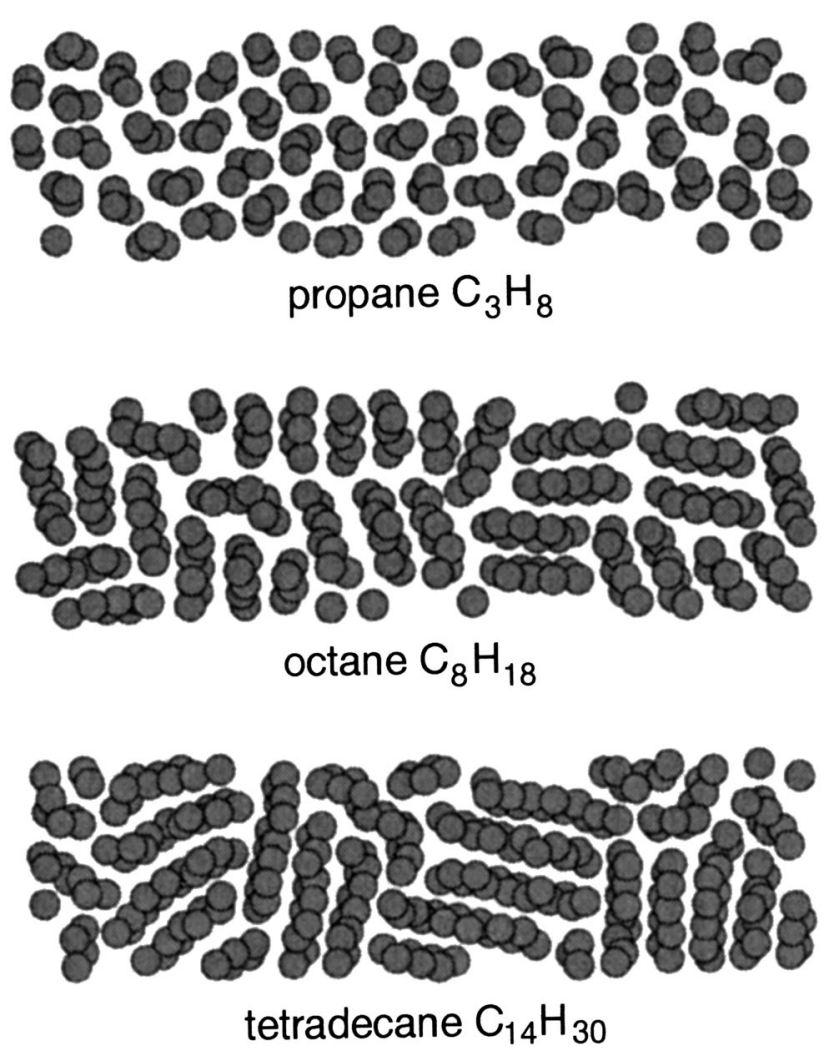

FIG. 11. Central sections of the block-substrate contacts (see Fig. 10), with only the lubricant molecules shown. The alkane lubricants in this figure are propane, octane, and tetradecane.

incommensurate surface structures are formed, making pinning effects much weaker.

We now address the question of why the long-chain alkanes are better boundary lubricants than the short-chain ones. We first study the lubricant film in the middle of the contact between the block and the substrate. This is done at a point where all the systems have one layer left between the surfaces and where they are at almost the same average pressure. These conditions are fulfilled when the block has moved $16 \AA$.

The central part of the system is shown in Fig. 10(a). The investigated section is shown in Fig. 10(b) where the wall atoms have been removed. The size of the section is $75.9 \AA$ by $20 \AA$ and it includes the middle of the contact area.

The central sections of three systems, propane, octane and tetradecane are shown in Fig. 11. By simple visual inspection of this figure, it is obvious that the lubricant molecules are covering an increasing part of the surface when going from propane, over octane, to tetradecane. We will define this property as the surface density of lubricant atoms, which is thus larger for tetradecane than for the other two alkanes.

The number of carbon atoms in the contact section when the block has moved $16 \AA$ was counted for all the seven alkanes, both in the case of pure squeezing and combined squeezing and sliding. In the latter case, the considered sections are centered about a line $16 \AA$ to the right of the middle of the contact shown in Fig. 10 as this is the distance the

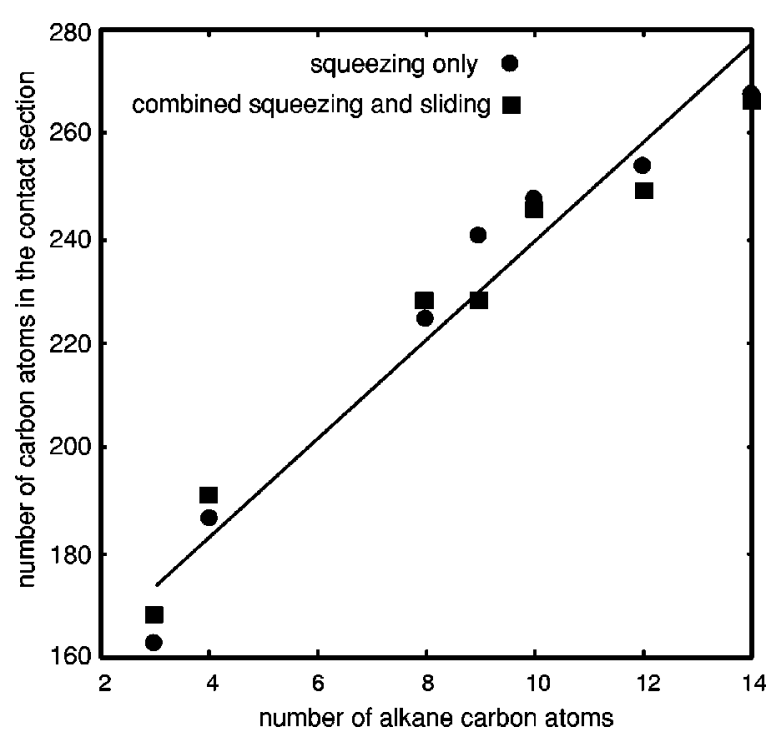

FIG. 12. The number of alkane carbon atoms in the contact section as a function of the length of the alkane. Only one correlation line is drawn as the squeezing and the combined squeezing and sliding cases are not significantly different.

block has moved horizontally during the motion. All these results are plotted in Fig. 12. This figure not only shows an increasing carbon-atom surface density for the longer alkanes but also that no significant differences are observed between the pure squeezing and the combined squeezing and sliding cases. This is in good correlation with the results from Figs. 7 and 9. The explanation of this increasing surface density of lubricant atoms with increasing length of the alkane can be found in the bead-bead distances. The atoms in an alkane molecule have very short bonds as compared to the van der Waals distances between the bead units of nearby molecules. This means that the contact section will have a larger number of the bead units when the lubricant is a long alkane, and, as we will now show, this implies a higher squeeze-out pressure for the long-chain alkanes.

It has been shown both theoretically ${ }^{18,19}$ and experimentally ${ }^{19-21}$ that the layering transition $n \rightarrow n-1$ start by a thermal fluctuation opening up a small "hole" in the lubrication film as indicated in Fig. 13 for the $n=1 \rightarrow 0$ transition. For a wetting liquid (as in the present case with hy-

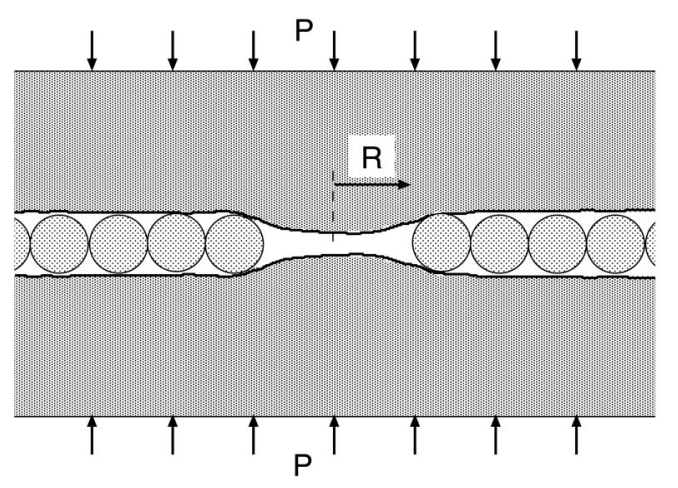

FIG. 13. The squeeze-out of the last lubricant monolayer from the contact area between two elastic solids. The squeeze-out is initiated by the formation of a small circular "hole" (radius typically $R \approx 10-15 \AA$ ). 


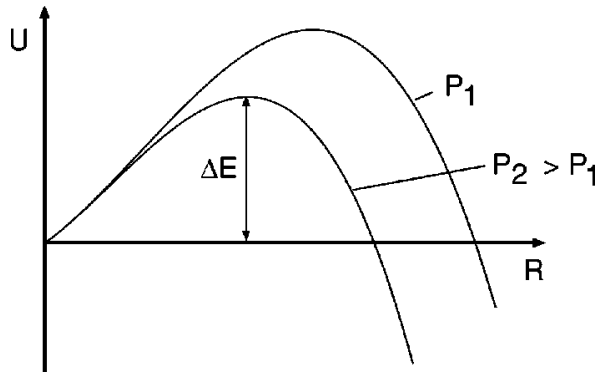

FIG. 14. The free energy $U(R)$ as a function of the radius $R$ of the hole. The barrier height $\Delta E$ decreases when the applied squeezing pressure $P$ increases.

drocarbons on metals or on metal oxides) the formation of a hole cost a wall-lubricant binding energy and also a line energy as a result of unsaturated bonds of the lubricant molecules towards the interior of the "hole." These (positive) energy terms scales as $\sim R^{2}$ and $\sim R$, respectively, with the radius $R$ of the hole. On the other hand, when the hole has been formed, the confining solid walls, which are squeezed together with a high pressure $P$, will relax inwards at the hole as illustrated in Fig. 13. This gives rise to a (negative) relaxation energy which scales as $\sim R^{3}$ with the radius of the hole (see the Appendix) so that the total energy is of the form

$$
U(R)=a R+b R^{2}-c R^{3},
$$

where $a, b$, and $c$ are positive numbers. One can show that $c \sim P^{2} / E$, where $E$ is the elastic modulus of the solid walls (see the Appendix). The function $U(R)$ has the form shown in Fig. 14. As the pressure $P$ increases the barrier $\Delta E$ decreases, and when the barrier becomes of order $\sim 1 \mathrm{eV}$ the system can, due to a thermal fluctuation, "jump" over the barrier in a macroscopic time period, say $1 \mathrm{~s}$.

The MD results presented above are easy to understand based on the nucleation model discussed above. The crucial observation is that the binding distance between the carbon atoms within the hydrocarbon chains $\left(r_{\mathrm{C}-\mathrm{C}} \approx 1.5 \AA\right)$ is much shorter than the van der Waals binding distance between the $\mathrm{CH}_{2}$ or $\mathrm{CH}_{3}$ bead units on different hydrocarbon chains, which is of order $r \approx 4 \AA$. This implies that as the hydrocarbon chain length increases, the number of bead units in direct contact with the solid walls will increase (see Fig. 12). Since the van der Waals lubricant-wall binding energy is roughly proportional to the number of bead units in contact with the wall, it follows that the hydrocarbon-wall binding energy per unit area increases with increasing chain length. At the same time the cohesive energy in the hydrocarbon liquid also increases, but at a lower rate than the hydrocarbon-wall binding energy, since the latter is usually much stronger than the binding between the hydrocarbon molecules. This is a consequence of the fact that most metals have much higher electron concentration than hydrocarbon liquids, and also more low-energy electronic excitations; both effects result in a stronger van der Waals interaction energy. Thus, as the hydrocarbon chain length increases, the coefficients $a$ and $b$ in Eq. (2) will both increase, resulting (for fixed $c$, i.e., for fixed applied pressure) in a higher activation barrier for squeeze-out. Hence, the pressure necessary in order to nucleate the layering transition $n=1 \rightarrow 0$ will increase with increasing chain length, in agreement with our MD computer simulations. Thus, long chain hydrocarbons are usually better boundary lubricants than short chain hydrocarbons, not only because of the increased viscosity which tend to increase the lubricant film thickness, butalso because the squeeze out of the last few monolayers (for which the viscosity is irrelevant and not even well defined), will, as described above, occur at higher pressure because of the better wetting (high spreading pressure) properties of the long-chain hydrocarbons. Using the theory presented in Ref. 18, the discussion presented above can be quantified, but already our qualitative discussion exhibits the basic physics and is sufficient for our purposes.

We now return to the experimental results from lubricity tests. With the results from the computer simulations in mind, are we now able to explain the wear scar diameter dependence on the fuel viscosity? The viscosity is a bulk property of a fluid. In the computer simulations we have molecular thin films of lubricant in a solidlike state for which the viscosity is not well-defined. But the results of the simulations indicate that the longer alkanes (with high viscosity) are better boundary lubricants than the shorther ones (with low viscosity), which correlates well with the wear test results. However, we do not believe that the viscosity (a bulk property) has any significant influence on the wear test results. The explanation is more likely that the longer molecules have a larger number of lubricant atoms in the block-substrate contact zone, resulting in a larger lubricant-wall binding energy per unit area, and a larger energy barrier for nucleating the squeeze-out (see above).

Real surfaces, in particular when they have been exposed to wear, will have surface roughness on many different length scales. Trapping of lubricant in the surface roughness "cavities" may result in a reduction of the wear. $^{22}$ In this case it is not clear if long chain or short chain hydrocarbons result in the strongest reduction of the wear. Our simulations show that, with a cosine roughness profile with a wavelength of $\lambda=38.9 \AA$, the long chain hydrocarbons result in the greater number of trapped bead units.

Recent wear testing ${ }^{11}$ also included branched lubricant molecules. The linear butane and the branched iso-butane have about the same viscosity, but gave significantly different wear scar diameters, corresponding to nearly 2 times higher wear volume for iso-butane. This supports our relation between the wear volume and the hydrocarbon chain length, rather than the viscosity, and indicates that the surface density of lubricant atoms can be altered by changing the molecule structure. Furthermore, computer simulations have shown that molecularly thin layers of branched hydrocarbons give rise to more disordered structures than linear chains, ${ }^{23,24}$ and that the pressure to nucleate squeeze-out decreases when disorder occurs in the lubricant film. ${ }^{14} \mathrm{We}$ will look into this aspect of boundary lubrication in the near future. 


\section{SUMMARY AND CONCLUSIONS}

We have studied the lubricating properties of seven linear alkanes with lengths varying from 3 carbon atoms (propane) to 14 carbons atoms (tetradecane). We have used a model which confines the alkanes between two approaching solids with long range elasticity. The results indicate that longer alkanes are better boundary lubricants than shorter ones, as the layering transitions occur at higher average pressure with increasing chain length. This results from the higher surface density of lubricant atoms in the contact zone for the longer alkanes, which gives a higher lubricant-wall binding energy per unit surface area (and a higher wetting ability). Physically, the inter-atomic bonds in the alkane molecules permit higher surface density as they are shorter than the van der Waals distances between the bead units of nearby molecules.

The observations in this work present new insight into boundary lubrication and the related wear, and seems to be able to explain the outcome of recent lubricity-wear tests involving linear and branched molecules.

\section{ACKNOWLEDGMENTS}

I.M.S. and V.N.S. acknowledge support from IFF, FZJülich, hospitality and help of the staff during their research visits. I.M.S. also acknowledges financial support from the European project AFforHD, the Otto Monsted foundation and the Civ. Ing. Kristian Rasmussen og Hustru Gunild Rasmussens foundation. B.N.J.P. thanks the EC for a "Smart Quasicrystals" grant under the EC Program "Promoting Competitive and Sustainable Growth."

\section{APPENDIX: ESTIMATION OF ELASTIC RELAXATION ENERGY}

In this Appendix we estimate the elastic relaxation energy resulting from the formation of a small hole in the lubrication film, see Fig. 13. Before the hole is formed, the stress in the solid at the interface is $\sigma=P$, where $P$ is the squeezing pressure. When the hole has been formed, thes- tress is reduced to nearly zero in a volume element $\sim R^{3}$. Thus the change in the elastic energy is of order

$$
\sim \int d^{3} x \sigma \epsilon \approx \frac{1}{E} \int d^{3} x \sigma^{2} \approx \frac{P^{2} R^{3}}{E}
$$

which enters as the last term in Eq. (2).

${ }^{1}$ B. N. J. Persson, Sliding Friction: Physical Principles and Applications (Springer, Heidelberg, 2000).

${ }^{2}$ J. N. Israelachvili, Intermolecular and Surface Forces (Academic, London, 1995).

${ }^{3}$ A. M. Homola, J. N. Israelachvili, P. M. McGuiggan, and M. L. Gee, Wear 136, 65 (1990).

${ }^{4}$ P. I. Lacey and S. A. Howell, Soc. Automot. Eng. [Spec. Publ.] SAE Tech. Paper 982567 (1998)

${ }^{5}$ Annual Book of ASTM Standards: D6079-97 5.03, 1355 (1998)

${ }^{6}$ ISO Standards, ISO 12156-1 and 2 (1997).

${ }^{7}$ I. M. Sivebaek and S. C. Sorenson, Soc. Automot. Eng. [Spec. Publ.] SAE Tech. Paper 2000-01-2970 (2000).

${ }^{8}$ P. I. Lacey and R. L. Mason, Soc. Automot. Eng. [Spec. Publ.] SAE Tech. Paper 2000-01-1917 (2000)

${ }^{9}$ D. P. Wei, H. A. Spikes, and S. Korcek, Tribol. Trans. 42, 4, 813 (1999).

${ }^{10}$ Handbook of Chemistry and Physics, 82th ed. (CRC, Boca Raton, 2001).

${ }^{11}$ I. M. Sivebaek, S. C. Sorenson, and J. Jakobsen (unpublished).

${ }^{12}$ I. M. Sivebaek, S. C. Sorenson, and J. Jakobsen, Soc. Automot. Eng. [Spec. Publ.] SAE Tech. Paper 2001-01-2013 (2001).

${ }^{13}$ B. N. J. Persson and P. Ballone, J. Chem. Phys. 112, 9524 (2000).

${ }^{14}$ B. N. J. Persson, V. N. Samoilov, S. Zilberman, and A. Nitzan, J. Chem. Phys. 117, 3897 (2002)

${ }^{15}$ W. I. Jorgensen, J. D. Madura, and C. J. Swenson, J. Am. Chem. Soc. 106, 6996 (1984)

${ }^{16}$ D. K. Dysthe, A. H. Fuchs, and B. Rousseau, J. Chem. Phys. 112, 7581 (2000).

${ }^{17}$ T. K. Xia, J. Ouyang, M. W. Ribarsky, and U. Landman, Phys. Rev. Lett. 69, 1967 (1992).

${ }^{18}$ B. N. J. Persson and E. Tosatti, Phys. Rev. B 50, 5590 (1994)

${ }^{19}$ S. Zilberman, T. Becker, F. Mugele, B. N. J. Persson, and A. Nitzan, J. Chem. Phys. (to be published).

${ }^{20}$ F. Mugele and M. Salmeron, Phys. Rev. Lett. 84, 5796 (2000).

${ }^{21}$ T. Becker and F. Mugele, J. Phys.: Condens. Matter 15, S321 (2003).

${ }^{22}$ U. Landman, W. D. Luedtke, and E. M. Ringer, Wear 153, 3 (1992).

${ }^{23}$ J. Gao, W. D. Luedtke, and U. Landman, J. Chem. Phys. 106, 4309 (1997).

${ }^{24}$ J. Gao, W. D. Luedtke, and U. Landman, J. Phys. Chem. B 101, 4013 (1997) 